\title{
Location for the Left Eigenvalues of Quaternionic Matrix
}

\author{
Xiangyun Chen \\ Department of Basic Sciences, Yancheng Institute of Technology \\ Yancheng 224051, China \\ E-mail: cxy808080@163.com
}

\begin{abstract}
The purpose of this paper is to locate and estimate the left eigenvalues of quaternionic matrices. We present some distribution theorems for the left eigenvalues of square quaternionic matrices based on the generalized Gerschgorin theorem and generalized Brauer theorem.
\end{abstract}

Keywords: Quaternion division algebra, Left eigenvalues, Generalized Gerschgorin theorem, Generalized Brauer theorem

\section{Subject Classification: AMS (2000) 15A21}

\section{Introduction and symbols}

Linear algebra over quaternion division algebra has drawn the attention of the researchers of mathematics, physics, and computer science widely. The research of matrices is continuously an important aspect of algebra problem over quaternion division algebra. Quaternion is mostly used in computer vision because of their ability to represent rotations in 4D spaces [Ran Ruisheng, 2006]. It is also playing an important role in quantum physics and geostatics and so forth [Wang Qinggui, 1983; Zhang Gangshu, 1982].

In this paper we shall mainly adopt the notation and terminology in [Fuzhen Zhang, 2007], for convenience, recall it. As usual, $R$ and $C$ are the sets of the real and complex numbers respectively. We denote by $H$ the set of real quaternions:

$$
H=\left\{a=a_{0}+a_{1} i+a_{2} j+a_{3} k, a_{0}, a_{1}, a_{2}, a_{3} \in R\right\}
$$

For $a=a_{0}+a_{1} i+a_{2} j+a_{3} k \in H$, the conjugate of $a$ is $\bar{a}=a^{*}=a_{0}-a_{1} i-a_{2} j-a_{3} k$ and the norm of $a$ is $|a|=\sqrt{a \bar{a}}=\sqrt{\bar{a} a}=$ $\left(a_{0}^{2}+a_{1}^{2}+a_{2}^{2}+a_{3}^{2}\right)^{1 / 2}$. Let $H^{n \times n}$ and $H^{n \times 1}$ be the collections of all $n \times n$ matrices with entries in $H$ and $n$-column vectors respectively. Let $I_{n}$ be the collections of all $n \times n$ unit matrices with entries in $H$. For $X \in H^{n \times 1}, X^{T}$ is the transpose of $X$. If $X=\left(x_{1}, x_{2}, \cdots, x_{n}\right)^{T}$, then $\bar{X}=\left(\bar{x}_{1}, \bar{x}_{2}, \cdots, \bar{x}_{n}\right)^{T}$ is the conjugate of $X$ and $X^{*}=\left(\bar{x}_{1}, \bar{x}_{2}, \cdots, \bar{x}_{n}\right)$ is the conjugate transpose of $X$. The norm of $X$ is defined to be $|X|=\sqrt{X^{*} X}$. For a $n \times n$ matrix $A=\left(a_{i j}\right)_{n \times n}\left(a_{i j} \in H\right)$, the conjugate transpose of $A$ is the $n \times n$ matrix $A^{*}=\overline{A^{T}}$.

\section{Some definitions and lemmas}

Before starting, we quickly review some basic definitions and lemmas.

Definition 2.1 (Junliang $\mathrm{Wu}, 2008$ ) Suppose that $a$ is a given real quaternion and is a positive real number. The set $G=\{z \in H:|z-a| \leq r\}$ is said to be a generalized spherical neighborhood with the center $a$ and the radius $r$.

Definition 2.2 (Fuzhen Zhang, 1997) Let $A \in H^{n \times n}$, a quaternion $\lambda$ is said to be left eigenvalue of $A$ if there exists nonzero quaternion column vector $X=\left\{x_{1}, x_{2}, \cdots, x_{n}\right\}^{T}$ such that $A X=\lambda X$.

Definition 2.3 (Fuzhen Zhang, 1997) Let $A \in H^{n \times n}$, a quaternion $\lambda$ is said to be right eigenvalue of $A$ if there exists nonzero quaternion column vector $Y=\left\{y_{1}, y_{2}, \cdots, y_{n}\right\}^{T}$ such that $A Y=Y \lambda$.

Lemma 2.1 (Fuzhen Zhang, 2007) (The generalized Gerschgorin Theorem) Let $A=\left(a_{i j}\right) \in H^{n \times n}$. Then all the left eigenvalues of $A$ are located in the union of $n$ generalized spherical neighborhoods

$$
\bigcup_{i=1}^{n}\left\{z \in H:\left|z-a_{i i}\right| \leq R_{i}\right\}
$$

where $R_{i}=\sum_{\substack{j=1 \\ j \neq i}}^{n}\left|a_{i j}\right|$.

Lemma 2.2 (Junliang Wu, 2008) (The generalized Gerschgorin Theorem) Let $A=\left(a_{i j}\right) \in H^{n \times n}$. Then all the eigenvalues of $A$ are located in the union of $n$ generalized spherical neighborhoods 


$$
\bigcup_{i=1}^{n}\left\{z \in H:\left|z-a_{i i}\right| \leq \sqrt{n-1} R_{i}\right\}
$$

Where $R_{i}=\sqrt{\sum_{\substack{j=1 \\ j \neq i}}^{n}\left|a_{i j}\right|^{2}}$

Lemma 2.3 (Junliang Wu, 2008). (The generalized Brauer Theorem) Let $A=\left(a_{i j}\right) \in H^{n \times n}$. Then all the left eigenvalues of $A$ are located in the union of $\frac{n(n-1)}{2}$ generalized oval neighborhoods

$$
\bigcup_{\substack{i, j=1 \\ i \neq j}}^{n}\left\{z \in H:\left|z-a_{i i}\right|\left|z-a_{j j}\right| \leq R_{i} R_{j}\right\}
$$

where $R_{i}=\sum_{\substack{j=1 \\ j \neq i}}^{n}\left|a_{i j}\right|$.

\section{The location for the left eigenvalues of quaternionic matrix}

Recently, the forms of the famous Gerschgorin Theorem and Brauer Theorem were studied over real quaternion division algebra (Fuzhen Zhang, 2007; Junliang Wu, 2008; Junliang Wu, 2008). They solved some distribution and estimation problems of left eigenvalues of quaternionic matrices. In this section, we will present some distribution theorems for the left eigenvalues of square quaternionic matrices based on the generalized Gerschgorin Theorem and Brauer Theorem.

Theorem 1. Let $A=\left(a_{i j}\right) \in H^{n \times n}$ and let $\alpha \in[0,1]$ be given. Then all the left eigenvalues of $A$ are located in the union of $n$ generalized spherical neighborhoods

$$
\lambda(A) \subset G(A)=\bigcup_{i=1}^{n}\left\{z \in H:\left|z-a_{i i}\right| \leq(n-1)^{\frac{1-\alpha}{2}} R_{i}^{\alpha} C^{1-\alpha_{i}}\right\},
$$

where $\alpha(A)$ is the set of the left eigenvalues of $\mathrm{A}, R_{i}=\sum_{\substack{j=1 \\ j \neq i}}^{n}\left|a_{i j}\right|, C_{i}=\sqrt{\sum_{\substack{j=1 \\ j \neq i}}^{n}\left|a_{i j}\right|^{2}}$

Proof. As the cases $\alpha=0$ and $\alpha=1$, by Lemma 2.1 and lemma 2.2, the result holds. Furthermore, we may assume that $R_{i}=\sum_{\substack{j=1 \\ j \neq i}}^{n}\left|a_{i j}\right|>0$, because we may perturb $A$ by inserting a small nonzero entry into any row in which $R_{i}=\sum_{\substack{j=1 \\ j \neq i}}^{n}\left|a_{i j}\right|=0$, the resulting matrix has an inclusion generalized spherical neighborhood that is larger than the generalized spherical neighborhood for $A$. And the result follows in the limit as the perturbation goes to zero. Now we suppose that $A X=\lambda X$ with $0 \neq X=\left(x_{1}, x_{2}, \cdots, x_{n}\right)^{T} \in H^{n \times 1}$. Then

$$
\sum_{j=1}^{n} a_{i j} x_{j}=\lambda x_{i}
$$

Let $\left|x_{i}\right| \geq\left|x_{j}\right|, i \neq j=1,2, \cdots, n$, then $x_{i} \neq 0$ and

$$
\left|\lambda-a_{i i}\right|\left|x_{i}\right|=\left|\sum_{\substack{j=1 \\ j \neq i}}^{n} a_{i j} x_{j}\right| \leq \sum_{\substack{j=1 \\ j \neq i}}^{n}\left|a_{i j}\right|\left|x_{j}\right|=\sum_{\substack{j=1 \\ j \neq i}}^{n}\left|a_{i j}\right|^{\alpha}\left(\left|a_{i j}\right|^{1-\alpha}\left|x_{j}\right|\right)
$$

Let $p=\frac{1}{\alpha}$ and $q=\frac{1}{1-\alpha}$, then $\frac{1}{p}+\frac{1}{q}=1$. By Holder's inequality (page 147), it is not hard to see the following

$$
\left|\lambda-a_{i i} \| x_{i}\right| \leq\left[\sum_{\substack{j=1 \\ j \neq i}}^{n}\left(\left|a_{i j}\right|^{\alpha}\right)^{\frac{1}{\alpha}}\right]^{\alpha}\left[\sum_{\substack{j=1 \\ j \neq i}}^{n}\left(\left|a_{i j}\right|^{1-\alpha}\left|x_{j}\right|\right)^{\frac{1}{1-\alpha}}\right]^{1-\alpha}=R_{i}^{\alpha}\left[\sum_{\substack{j=1 \\ j \neq i}}^{n}\left(\left|a_{i j}\right|^{1-\alpha}\left|x_{j}\right|\right)^{\frac{1}{1-\alpha}}\right]^{1-\alpha}
$$

Since $R_{i}=\sum_{\substack{j=1 \\ j \neq i}}^{n}\left|a_{i j}\right|>0$, we can obtain

$$
\frac{\left|\lambda-a_{i i}\right|\left|x_{i}\right|}{R_{i}^{\alpha}} \leq\left[\sum_{\substack{j=1 \\ j \neq i}}^{n}\left(\left|a_{i j}\right|^{1-\alpha}\left|x_{j}\right|\right)^{\frac{1}{1-\alpha}}\right]^{1-\alpha},
$$


that is

$$
\left(\frac{\left|\lambda-a_{i i}\right|\left|x_{i}\right|}{R_{i}^{\alpha}}\right)^{\frac{1}{1-\alpha}} \leq \sum_{\substack{j=1 \\ j \neq i}}^{n}\left(\left|a_{i j}\right|^{1-\alpha}\left|x_{j}\right|\right)^{\frac{1}{1-\alpha}}=\sum_{\substack{j=1 \\ j \neq i}}^{n}\left|a_{i j}\right|\left|x_{j}\right|^{\frac{1}{1-\alpha}}
$$

i.e.

$$
\left(\frac{\left|\lambda-a_{i i}\right|}{R_{i}^{\alpha}}\right)^{\frac{1}{1-\alpha}}\left|x_{i}\right|^{\frac{1}{1-\alpha}} \leq \sum_{\substack{j=1 \\ j \neq i}}^{n}\left|a_{i j}\right|\left|x_{j}\right|^{\frac{1}{1-\alpha}}
$$

Using Cauchy-Schwarz inequality (Junliang Wu, 2008), we have

$$
\left(\frac{\left|\lambda-a_{i i}\right|}{R_{i}^{\alpha}}\right)^{\frac{1}{1-\alpha}}\left|x_{i}\right|^{\frac{1}{1-\alpha}} \leq \sum_{\substack{j=1 \\ j \neq i}}^{n}\left|a_{i j}\right|\left|x_{j}\right|^{\frac{1}{1-\alpha}} \leq \sqrt{\sum_{\substack{j=1 \\ j \neq i}}^{n}\left|a_{i j}\right|^{2}} \sqrt{\sum_{\substack{j=1 \\ j \neq i}}^{n}\left|x_{j}\right|^{\frac{2}{1-\alpha}}} .
$$

Since $\left|x_{i}\right| \geq\left|x_{j}\right|, i \neq j=1,2, \cdots, n$, it follows that

$$
\left(\frac{\left|\lambda-a_{i i}\right|}{R_{i}^{\alpha}}\right)^{\frac{1}{1-\alpha}}\left|x_{i}\right|^{\frac{1}{1-\alpha}} \leq \sum_{\substack{j=1 \\ j \neq i}}^{n}\left|a_{i j}\right|\left|x_{j}\right|^{\frac{1}{1-\alpha}} \leq \sqrt{\sum_{\substack{j=1 \\ j \neq i}}^{n}\left|a_{i j}\right|^{2}} \cdot \sqrt{(n-1)}\left|x_{i}\right|^{\frac{1}{1-\alpha}}
$$

and therefore

$$
\left(\frac{\left|\lambda-a_{i i}\right|}{R_{i}^{\alpha}}\right)^{\frac{1}{1-\alpha}} \leq \sqrt{(n-1) \sum_{\substack{j=1 \\ j \neq i}}^{n}\left|a_{i j}\right|^{2}}=\sqrt{(n-1)} C_{i}
$$

Thus, $\left|\lambda-a_{i i}\right| \leq(n-1)^{\frac{1-\alpha}{2}} R_{i}^{\alpha} C_{i}^{1-\alpha}$ for some $i$, that is, $\lambda$ lies in a generalized spherical neighborhood around $a_{i i}$ of radius $(n-1)^{\frac{1-\alpha}{2}} R_{i}^{\alpha} C_{i}^{1-\alpha}$. Since we do not know which $i$ is appropriate to each left eigenvalue $\lambda$ (unless we know the associated eigenvector, in which case we would know $\lambda$ exactly and would not be interested in locating it), we can only conclude that $\lambda$ lies in the union of $n$ generalized spherical neighborhoods. Then the theorem is proved. $\square$

Theorem 2. Let $A=\left(a_{i j}\right) \in H^{n \times n}$. Then all the left eigenvalues of $A$ are located in the union of $\frac{n(n-1)}{2}$ generalized oval neighborhoods

$$
\lambda(A) \subset G(A)=\bigcup_{\substack{i, j=1 \\ i \neq j}}^{n}\left\{z \in H:\left|z-a_{i i}\right|\left|z-a_{j j}\right| \leq(n-1) R_{i} R_{j},\right.
$$

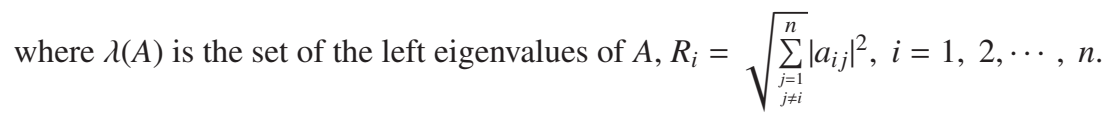

Proof. Let $\lambda$ be a left eigenvalue of $A$, then

$$
A X=\lambda X,
$$

where $0 \neq X=\left(x_{1}, x_{2}, \cdots, x_{n}\right)^{T} \in H^{n \times 1}$ is an eigenvector of $A$ corresponding to $\lambda$. If we let $\left|x_{p}\right|=|\max | x_{i} \mid, i=$ $1,2, \cdots, n$, then $x_{p} \neq 0$. Obviously, if the other entries of quaternion vector $X$ are zero, then the result holds naturally. If there are at least two entries of vector $X$ are nonzero, then we let $\left|x_{p}\right|=\max \left|x_{i}\right|$ and $\left|x_{q}\right|=\max \left|x_{i}\right|, i \neq p$. So, we have $x_{p} \neq 0, x_{q} \neq 0$ and $\left|x_{p}\right| \geq\left|x_{q}\right| \geq\left|x_{i}\right|$. Since $A X=\lambda X$, then

$$
\sum_{j=1}^{n} a_{p j} x_{j}=\lambda x_{p}
$$

Using Cauchy-Schwarz inequality (Junliang Wu, 2008), we have

$$
\left|\lambda-a_{p p}\right|=\left|\frac{\sum_{j \neq p} a_{p j} x_{j} \bar{x}_{p}}{\left|x_{p}\right|^{2}}\right| \leq \sqrt{\sum_{j \neq p}\left|a_{p j}\right|^{2}} \cdot \sqrt{\sum_{j \neq p}\left|\frac{x_{j}}{\left|x_{p}\right|}\right|^{2}\left|\frac{\overline{x_{p}}}{\left|x_{p}\right|}\right|^{2}} \leq \sqrt{(n-1) \sum_{j \neq p}\left|a_{p j}\right|^{2}}\left|\frac{x_{q}}{x_{p}}\right|=\sqrt{n-1} R_{p}\left|\frac{x_{q}}{x_{p}}\right| .
$$

Meanwhile, we also have

$$
\left|\lambda-a_{q q}\right|=\left|\frac{\sum_{j \neq q} a_{q j} x_{j} \bar{x}_{q}}{\left|x_{q}\right|^{2}}\right| \leq \sqrt{\sum_{j \neq}\left|a_{q j}\right|^{2}} \cdot \sqrt{\sum_{j \neq q}\left|\frac{x_{j}}{\left|x_{q}\right|}\right|^{2}\left|\frac{\overline{x_{q}}}{\left|x_{q}\right|}\right|^{2}} \leq \sqrt{(n-1) \sum_{j \neq q}\left|a_{q j}\right|^{2}}\left|\frac{x_{p}}{x_{q}}\right|=\sqrt{n-1} R_{q}\left|\frac{x_{p}}{x_{q}}\right|
$$


Taking the produce of (3.1) and (3.2) permits us to eliminate the unknown ratios of component of $x$ and obtain

$$
\left|\lambda-a_{p p}\right|\left|\lambda-a_{q q}\right| \leq \sqrt{n-1} R_{p}\left|\frac{x_{q}}{x_{p}}\right| \cdot \sqrt{n-1} R_{q}\left|\frac{x_{p}}{x_{q}}\right|=(n-1) R_{p} R_{q}
$$

Thus, all the left eigenvalues of $A$ are located in the union of $\frac{n(n-1)}{2}$ generalized oval neighborhoods. Then the proof is completed. $\square$

Theorem 3. Let $A=\left(a_{i j}\right) \in H^{n \times n}$. Then all the left eigenvalues of $A$ are located in the union of $\frac{n(n-1)}{2}$ generalized oval neighborhoods

$$
\lambda(A) \subset G(A)=\bigcup_{\substack{i, j=1 \\ i \neq j}}\left\{z \in H:\left|z-a_{i i}\right|\left|z-a_{j j}\right| \leq R_{i}\left(R_{i}+\left|a_{i i}-a_{j j}\right|\right)\right\}
$$

where $\lambda(A)$ is the set of the left eigenvalues of $A, R_{i}=\sum_{\substack{j=1 \\ j \neq i}}^{n}\left|a_{i j}\right|,(1 \leq i, j \leq n)$.

Proof. Let $\lambda$ be a left eigenvalue of $A$, then

$$
A X=\lambda X
$$

where $0 \neq X=\left(x_{1}, x_{2}, \cdots, x_{n}\right)^{T} \in H^{n \times 1}$ is an eigenvector of $A$ corresponding to $\lambda$. By Lemma 2.1, we have

$$
\left|\lambda-a_{i i}\right| \leq \sum_{\substack{j=1 \\ j \neq i}}^{n}\left|a_{i j}\right|=R_{i}
$$

so

$$
\left|\lambda-a_{j j}\right|=\left|\lambda-a_{i i}+a_{i i}-a_{j j}\right| \leq\left|\lambda-a_{i i}\right|+\left|a_{i i}-a_{j j}\right| \leq R_{i}+\left|a_{i i}-a_{j j}\right|
$$

Then, we can obtain

$$
\left|\lambda-a_{i i}\right|\left|\lambda-a_{j j}\right| \leq R_{i}\left(R_{i}+\left|a_{i i}-a_{j j}\right|\right), i \neq j,
$$

therefore

$$
\lambda(A) \subset G(A)
$$

The proof is completed.

Example. Let

$$
A=\left(\begin{array}{ccc}
1 & i & j \\
i+\sqrt{3} k & 2 & 2 k \\
1+i+j+k & i+j+\sqrt{14} k & 3
\end{array}\right) .
$$

By generalized Brauer Theorem (Junliang Wu, 2008), we can obtain that all the left eigenvalues of $A$ are located in the union of following generalized oval neighborhoods

$$
\{z \in H:|z-1| \cdot|z-2| \leq 8\} \cup\{z \in H:|z-1| \cdot|z-3| \leq 12\} \cup\{z \in H:|z-2| \cdot|z-3| \leq 24\} .
$$

By Theorem 3, we can obtain that all the left eigenvalues of are located in the union of following generalized oval neighborhoods

$$
\{z \in H:|z-1| \cdot|z-2| \leq 6\} \cup\{z \in H:|z-1| \cdot|z-3| \leq 8\} \cup\{z \in H:|z-2| \cdot|z-3| \leq 20\} .
$$

It is clear that the result of Theorem 3 is sharper than the generalized Brauer Theorem's. Therefore, Theorem 3 can obtain a good estimation for some quaternion matrices.

Theorem 4. Let $A=\left(a_{i j}\right) \in H^{n \times n}$. Then all the left eigenvalues of $A$ are located in the union of $\frac{n(n-1)}{2}$ generalized oval neighborhoods

$$
\lambda(A) \subset G(A)=\bigcup_{\substack{i, j=1 \\ i \neq j}}^{n}\left\{z \in H:\left|z-a_{i i}\right|\left|z-a_{j j}\right| \leq \sqrt{n-1} R_{i}\left(\sqrt{n-1} R_{i}+\left|a_{i i}-a_{j j}\right|\right)\right\},
$$

where $\lambda(A)$ is the set of the left eigenvalues of $A, R_{i}=\sqrt{\sum_{\substack{j=1 \\ j \neq i}}^{n}\left|a_{i j}\right|^{2}}, i=1,2, \cdots, n$.

Proof. According to the proof method of Theorem 3, we can prove Theorem 4, so we leave it to readers. 
Theorem 5. Let $A=\left(a_{i j}\right) \in H^{n \times n}$ and let $\lambda$ be a left eigenvalue of $A$ that is a boundary point of $\bigcup_{i=1}^{n}\left\{z \in H:\left|z-a_{i i}\right| \leq R_{i}\right\}$.

Let $A X=\lambda X$ with $X=\left(x_{i}\right) \neq 0$, and suppose $p$ is an index such that $\left|x_{p}\right|=\max _{1 \leq i \leq n}\left|x_{i}\right| \neq 0$. Then

(1) If $k$ is any index such that $\left|x_{k}\right|=\left|x_{p}\right|=\max _{1 \leq i \leq n}\left|x_{i}\right| \neq 0$, then $\left|\lambda-a_{k k}\right|=R_{k}$, that is the $k$ th generalized spherical neighborhood passes through $\lambda$.

(2) If $\left|x_{k}\right|=\left|x_{p}\right|=\max _{1 \leq i \leq n}\left|x_{i}\right| \neq 0$ for some $k=1,2, \cdots, n$ and if $a_{k j} \neq 0$ for some $j \neq k$, then $\left|x_{j}\right|=\left|x_{p}\right|=\max _{1 \leq i \leq n}\left|x_{i}\right| \neq 0$.

Proof. Let $A X=\lambda X$ with $X=\left(x_{i}\right) \neq 0$. We have

$$
\left(\lambda-a_{i i}\right) x_{i}=\sum_{\substack{j=1 \\ j \neq i}}^{n} a_{i j} x_{j}
$$

for all $i=1,2, \cdots, n$ and hence

$$
\left|\lambda-a_{i i}\right|\left|x_{i}\right|=\left|\sum_{\substack{j=1 \\ j \neq i}}^{n} a_{i j} x_{j}\right| \leq \sum_{\substack{j=1 \\ j \neq i}}^{n}\left|a_{i j}\right|\left|x_{j}\right| \leq \sum_{\substack{j=1 \\ j \neq i}}^{n}\left|a_{i j}\right|\left|x_{p}\right|=R_{i}\left|x_{p}\right| .
$$

Therefore, if $k$ is any index such that $\left|x_{k}\right|=\left|x_{p}\right|=\max _{1 \leq i \leq n}\left|x_{i}\right| \neq 0$, we must have $\left|\lambda-a_{k k}\right| \leq R_{k}$. But the condition is that $\left|\lambda-a_{k k}\right| \geq R_{k}$ for all $i=1,2, \cdots, n$, so we must actually have equality in both of (3.3) for $i=k$, that is

$$
\left|\lambda-a_{k k}\right|\left|x_{k}\right|=\left|\sum_{\substack{j=1 \\ j \neq i}}^{n} a_{k j} x_{j}\right|=\sum_{\substack{j=1 \\ j \neq k}}^{n}\left|a_{k j}\right|\left|x_{j}\right|=\sum_{\substack{j=1 \\ j \neq k}}^{n}\left|a_{k j}\right|\left|x_{k}\right|=R_{k}\left|x_{k}\right| .
$$

Since $\left|x_{k}\right|=\left|x_{p}\right|=\max _{1 \leq i \leq n}\left|x_{i}\right|>0$, assertion (1) follows from the identity (3.4). For assertion (2), by (3.4), we have

$$
\sum_{\substack{j=1 \\ j \neq k}}^{n}\left|a_{k j}\right|\left|x_{j}\right|=\sum_{\substack{j=1 \\ j \neq k}}^{n}\left|a_{k j} \| x_{k}\right|
$$

that is

$$
\sum_{\substack{j=1 \\ j \neq k}}^{n}\left|a_{k j}\right|\left(\left|x_{j}\right|-\left|x_{k}\right|\right)=0
$$

Then assertion (2) holds and the proof is completed.

Theorem 6. Let $A=\left(a_{i j}\right) \in H^{n \times n}$, and let $\lambda$ be a left eigenvalue of $A$ that is a boundary point of $\bigcup_{i=1}^{n}\left\{z \in H:\left|z-a_{i i}\right| \leq R_{i}\right.$. Suppose that all the entries of $A$ are nonzero. Then

(1) Every generalized spherical neighborhood of $A$ passes through $\lambda$.

(2) If $A X=\lambda X$ with $X=\left(x_{i}\right) \neq 0$, then $\left|x_{i}\right|=\left|x_{j}\right|$ for all $i, j=1,2, \cdots, n$.

Proof. By (1) of Theorem 5, we have that the $k$ th generalized spherical neighborhood passes through $\lambda$. Since all the entries of $A$ are nonzero, by (2) of Theorem 5, we have

$$
\left|x_{j}\right|=\left|x_{p}\right|=\max _{1 \leq i \leq n}\left|x_{i}\right| \neq 0
$$

for all $j=1,2, \cdots, n$. So every generalized spherical neighborhood of $A$ passes through $\lambda$.

Corollary 1. Let $A=\left(a_{i j}\right) \in H^{n \times n}$, and suppose that all the entries of $A$ are nonzero. If $\left|a_{i i}\right| \geq \sum_{\substack{j=1 \\ j \neq i}}^{n}\left|a_{i j}\right|$ for all $i=1,2, \cdots, n$ and if $\left|a_{i i}\right|>\sum_{\substack{j=1 \\ j \neq i}}^{n}\left|a_{i j}\right|$ for at least one value of $i=1,2, \cdots, n$, then $\lambda \neq 0$.

Proof. Let $\lambda$ be a left eigenvalue of $A$, and suppose that $\lambda=0$. Since $\left|a_{i i}\right| \geq \sum_{\substack{j=1 \\ j \neq i}}^{n}\left|a_{i j}\right|$ for all $i=1,2, \cdots, n, 0$ can't be an interior point of $\bigcup_{i=1}^{n}\left\{z \in H:\left|z-a_{i i}\right| \leq R_{i}\right.$ and hence $\lambda=0$ is a boundary point of $\bigcup_{i=1}^{n}\left\{z \in H:\left|z-a_{i i}\right| \leq R_{i}\right\}$. By Theorem 
6, we have that every generalized spherical neighborhood of $A$ passes through $\lambda$. But the condition is $\left|a_{i i}\right|>\sum_{\substack{j=1 \\ j \neq i}}^{n}\left|a_{i j}\right|$ for at least one value of $i=1,2, \cdots, n$, it's a contradiction, so $\lambda \neq 0$.

\section{References}

Fuzhen Zhang. (1997). Quaternions and matrices of quaternions. Linear Algebra Appl., 251, 21-57.

Fuzhen Zhang. (2007). Gersgorin type theorems for quaternionic matrices. Linear. Algebra. Appl., 424, 139-153.

Jiang Tongsong, Chen Li. (1999). Generalized diagonalization of matrices over quaternion Field (in Chinese). Applied Mathematics and Mechanics, (11)1203-1210.

Junliang Wu, Limin Zou, Xiangping Chen, Shengjie Li. (2008). The estimation of eigenvalues of sum, difference, and tensor product of matrices over quaternion division algebra. Linear .Algebra. Appl., 428, 3023-3033.

Junliang Wu. (2008). Distribution and Estimation for Eigenvalues of Real Quaternion Matrices. Computers and Mathematics with Applications, 55, 1998-2004.

Liping Huang. (2000). On two questions about quaternion matrices. Linear. Algebra. Appl., 318, 79-86.

Liping Huang. (2001). On left eigenvalues of a quaternionic matrix. Linear .Algebra. Appl., 323, 105-116.

Qingwen Wang. (2005). The General Solution to a system of real quaternion matrix equation. Computers and Mathematics with Applications, 49, 665-675.

Ran Ruisheng, Huang Tingzhu. (2006). The recognition of color Images based on the singular value decompositions of quaternion matrices (in Chinese). Computer Science, (7)227-229.

Wang Qinggui. (1983). Quaternion transformation and its application on the display-cement analysis of spatial mechanisms (in Chinese). Acta Mechanica Sinica, (01)54-61.

Zhang Gangshu. (1982). Commutatively of composition for finite rotations of a rigid body (in Chinese). Acta Mechanica Sinica, (04)363-368.

Zhuang Wajin. (2006). The guide of quaternion algebra theory (in Chinese). Science press, Beijing. 